EPJ manuscript No.

(will be inserted by the editor)

\title{
Sudden Critical Current Drops Induced in S/F Structures
}

\author{
Edgar J. Patiño ${ }^{1,2}$, Chris Bell ${ }^{1}$, and Mark G. Blamire ${ }^{1}$ \\ 1 Materials Science Department, IRC in Superconductivity and IRC in Nanotechnology, University of Cambridge, Cambridge, \\ United Kingdom \\ ' 2 Departamento de Física, Grupo de Fsica de la Materia Condensada, Universidad de los Andes, Bogotá, Colombia. \\ Received: date / Revised version: date
}

\begin{abstract}
In the search for new physical properties of $\mathrm{S} / \mathrm{F}$ structures, we have found that the superconductor critical current can be controlled by the domain state of the neighboring ferromagnet. The superconductor is a thin wire of thickness $d_{s} \approx 2 \xi_{S}$. Nb/Co and $\mathrm{Nb} / \mathrm{Py}$ (Permalloy $\mathrm{Ni}_{80} \mathrm{Fe}_{20}$ ) bilayer structures were grown with a significant magnetic anisotropy. Critical current measurements of $\mathrm{Nb} / \mathrm{Co}$ structures with ferromagnet thickness $d_{F}>30 \mathrm{~nm}$ show sudden drops in two very defined steps when the measurements are made along the hard axes direction (i.e. current track parallel to hard anisotropy axes direction). These drops disappear when they are made along the easy axis direction or when the ferromagnet thickness is below $30 \mathrm{~nm}$. The drops are accompanied by vortex flux flow. In addition magnetorestistance measurements close to $T_{C}$ show a sharp increase near saturation fields of the ferromagnet. Similar results are reproduced in Nb/Py bilayer structure with the ferromagnet thickness $d_{F} \sim 50 \mathrm{~nm}$ along the easy anisotropy axes. These results are explained as being due to spontaneous vortex formation and flow induced by Bloch domain walls of the ferromagnet underneath. We argue these Bloch domain walls produce a $2 \mathrm{D}$ vortex-antivortex lattice structure.
\end{abstract}

PACS. 72.25.Sv Critical Currents - 72.25.Qt Vortex lattices, flux pinning, flux creep - 72.25.Ha Magnetic Properties

\section{Introduction}

The proximity effect in superconductor (S) ferromagnet (F) bilayer structures has been subject of intensive research in the past ten years. This has been specially due to the effect exchange field has on Cooper pairs generating the so called Fulde Ferrel Larkin Ovchinnilov (FFLO) state. In this state the real part of the order parameter penetrates the ferromagnet and oscillates in proximity to the interface leading to the theoretically predicted oscillations of $T_{C}$ as a function of the ferromagnet thickness [1] and the experimentally verified $\pi$ phase shift in S/F multilayers 2, 3.

In addition to these investigations, other experiments carried out by Kinsey et al. [4 studied the effect that domain wall formation has on superconductivity. These were carried out on $\mathrm{Nb} / \mathrm{Co}$ bilayers, with $15-65 \mathrm{~nm}$ of $\mathrm{Nb}$ on $54 \pm 9 \mathrm{~nm}$ of Co, by critical current measurements of patterned films using photolithography. The geometry of the mask used for this process included the voltage contacts perpendicular to the current track, that leaded to stray fields on the current track. In these earlier studies an increase in the critical current measurements of the patterned wire was found by applying a magnetic field of the order of the coercive field of the ferromagnet. This

Send offprint requests to: Edgar J. Patiño effect was attributed to a reduction of the average exchange field sampled by the Cooper pairs within the domain wall giving rise to an enhancement of superconductivity so called domain wall superconductivity (DWS) [5]. However, in these experiments the effect of stray fields, from the mask geometry used, in the measurements could not be completely eliminated. Later a similar effect was reported in $\mathrm{Nb} / \mathrm{CuNi}$ trilayers by Rusanov et al. [6] again in this case the stray fields from the mask geometry were present (i.e. magnetic voltage contacts perpendicular to the current track). In an experiment, by the same authors [7, on $\mathrm{Nb} / \mathrm{Py}$ (Permalloy $\mathrm{Ni}_{80} \mathrm{Fe}_{20}$ ), with $21 \mathrm{~nm}$ of $\mathrm{Nb}$ and $20 \mathrm{~nm}$ of Py, suggests evidence of DWS. In this experiment Rusanov et al. found enhancement of superconductivity at coercive fields of the ferromagnet, by measuring magnetoresistance at the transition region between normal and superconducting state. Dips in magneto resistance measurements at coercive fields were attributed to be evidence of this effect. Nevertheless this effect was only found for large samples $(0.5 \mathrm{~mm} \times 4 \mathrm{~mm})$ and absent in small samples $(1.5 \mu \mathrm{m} \times 20 \mu \mathrm{m})$, where the authors argued that no stable domains form in small samples. In other experiments on $\mathrm{Nb} / \mathrm{CuNi}$ bilayers performed by Ryazanov et al. [8], they found magnetoresistive peaks in differential resistance measurements at coercive field of the ferromagnet. They explained this observations by spontaneous vortex 
Edgar J. Patiño, Chris Bell,, Mark G. Blamire: Sudden Critical Current Drops Induced in S/F Structures

formation and flow from stray fields from the domain walls of the ferromagnet. A theoretical work has been published by Burmistrov et al. 9 investigating the effect of Bloch domain walls on the current distribution in the superconductor. They also determine a lower critical value of domain wall magnetization above which vortex formation is favorable. Based on this idea experiments, performed by Steiner et al. 10 on $\mathrm{Fe} / \mathrm{Nb}$ and $\mathrm{Co} / \mathrm{Nb}$ bilayers, found a reduction of $T_{C} \approx 0.5 \%$ as a function of magnetic state of the ferromagnet. Additionally Bell et al. 11 have found a double peak structure in magnetoresistance measurements of MoGe/GdNi bilayered structures as a function of the magnetic state of the ferromagnet attributed to vortex formation and flux flow.

The aim of the present work is to further investigate the influence of Néel and Bloch domain walls in the superconducting pairing and vortex generation in $\mathrm{S} / \mathrm{F}$ hybrids. This investigation differs from previous ones in that it considers a wide range of ferromagnet's thickness and eliminates stray fields from the mask geometry. We also used Co and Py as ferromagnets where the domain wall structures is fairly well understood at least at room temperature.

\section{Critical Current and Magneto-Resistance Measurements}

In our experiment we have grown $\mathrm{Nb} / \mathrm{Co}$ and $\mathrm{Nb} / \mathrm{Py}$ bilayers (Nb on top) on $\mathrm{Si}$ (100) substrates using a UHV DC-magnetron sputtering system in a chamber cooled to $-100^{\circ} \mathrm{C}$ using liquid nitrogen. The base pressure was less than $3 \times 10^{-9}$ mbar. The partial oxygen base pressure measured using a mass spectrometer showed a value less than $0.1 \times 10^{-10}$ mbar. The Nb, Co and Py sputtering targets were $99.95 \%$ pure, deposited under Ar pressure of $0.5 \mathrm{~Pa}$, in an in-plane magnetic field $\mathrm{H}$ of approximately $400 \mathrm{Oe}$. Under these conditions the $\mathrm{F}$ layer in the bilayer structures grown had a strong magnetic anisotropy showing easy and hard axes anisotropy and coercive fields of about 35 and 16 Oe for the Co and 4.5 and 1.2 Oe for Py respectively. Several bilayer structures with Nb thickness $d_{N b}=25 \mathrm{~nm}$ (i.e. of twice the superconducting low temperature coherence length, $\xi_{S} \approx 12 \mathrm{~nm}$ ) and Co thickness, $d_{C o}$, between 2 and $50 \mathrm{~nm}$ were made where $d_{C o} \gg \xi_{C o} \approx 0.3 \mathrm{~nm}$, the ferromagnet's coherence length. The interface RMS roughness, measured using AFM, was about $0.3 \mathrm{~nm}$. The superconducting transition temperature $T_{C}$ for these $\mathrm{Nb} / \mathrm{Co}$ bilayer structures determined by four point resistivity measurements had an average value of $6.4 \pm 1 \mathrm{~K}$ depressed by the proximity effect, and a small transition width of 100 $\mathrm{mK}$ demonstrating the very high uniformity and quality of our films.

In order to compare the results the study was finalized by replacing the Co layer with Py with a thickness of $50 \mathrm{~nm}$. For this thickness all the results obtained for Co and discussed in this paper reproduce qualitatively the same for $\mathrm{Py}$.

Critical current measurements were made on bilayer structures patterned into tracks. Usually most experimen- tal configurations for doing four point measurements of narrow tracks include; the voltage contacts within the same structure and current tracks with sharp edges. Due to the presence of the ferromagnet in the S/F hybridstructures the free surfaces from, the voltage contacts or sharp edges, perpendicular to the ferromagnet's magnetization form free magnetic poles which effectively act as "external magnets". Depending upon the geometry and type of ferromagnet used these source of stray fields affect the superconductor to a lower or greater extent.

The current track was patterned using a mask without sharp edges at the center, where the measurements are made. The shape of the mask should mean that if there are free poles they contribute very little stray fields to the measurement section of the track. The voltage is measured using non magnetic $\mathrm{Cu}$ contacts. These prevent the wires acting as "external magnets". The center of the mask used to make the device is shown in Fig. 1. The geometry of the designed mask for the photolithography consists of three different current tracks of 2,5 and $7 \mu \mathrm{m}$ wide (drawn in blue color). This allowed us to take measurements with different aspect ratios. Only further away from the center the tracks width gradually increases in size to avoid sharp edges. Non magnetic $\mathrm{Cu}$ contacts (black color), spaced $\approx 7 \mu \mathrm{m}$, were deposited by sputter deposition in order to measure the voltage. This eliminates stray fields from the voltage contacts. The $\mathrm{Cu}$ contacts also play an important role in dissipating the heat generated by the Nb track in the normal state.

Current voltage (I-V) characteristics have been obtained. A plot of typical I-V characteristics at different temperatures is shown in Fig. 2. A clear jump from the normal to the superconducting state determines the value of the critical current $\left(I_{d p}\right)$. The small voltage onset below $I_{d p}$ is due to vortex motion, unavoidable close to $T_{C}$.

First $\mathrm{Nb} / \mathrm{Co}$ bilayers were patterned into tracks along the hard axis direction of the ferromagnet and then inplane critical current measurements were made with the field parallel to the track. Between a Cobalt thickness of $2 \mathrm{~nm}$ and $30 \mathrm{~nm}$ no appreciable changes in critical current in $\mathrm{Nb} / \mathrm{Co}$ bilayer structures were observed. However, for a ferromagnet thickness $d_{F} \geq 30 \mathrm{~nm}$ critical current measurements exhibit sudden drops at fields of the order of the coercive and saturation fields. The dips observed on a $25 \mathrm{~nm} \mathrm{Nb}$ on $42 \mathrm{~nm}$ Co, depicted in Fig. 3, show up to a $50 \%$ reduction from its maximum value. The drops increase in magnitude with temperature and thickness of the ferromagnet. However when taking the same measurements either with the field perpendicular to the track or by patterning the track along the easy axis direction the dips disappeared. This indicates a strong correlation with the anisotropy axes of the Cobalt.

Finally magnetoresistance measurements were made after stabilizing the temperature near $T_{C}$ at the transition region just above the zero resistance. A sudden increase in magnetoresistance as the one depicted in Fig. 4 for a sample of $25 \mathrm{~nm} \mathrm{Nb}$ on $50 \mathrm{~nm} \mathrm{Py}(\mathrm{Nb}(25) / \mathrm{Py}(50))$, is observed at the same field range for each of the samples where the dips occur. Note that in our experiments both; critical 


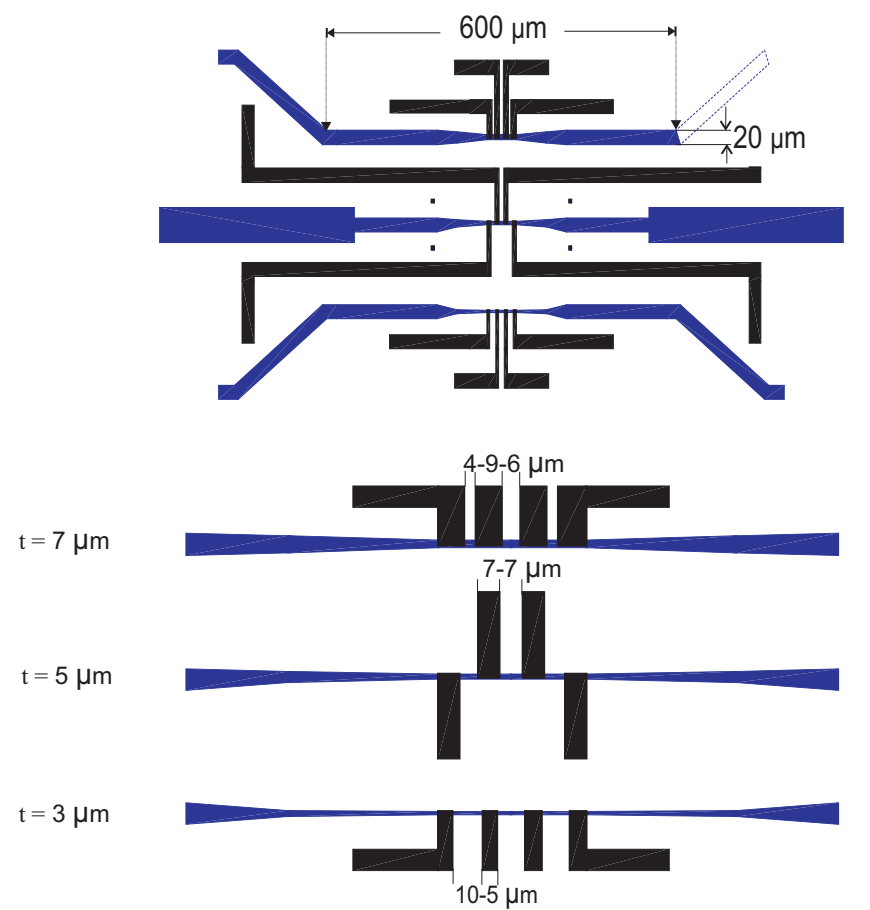

Fig. 1. Diagram of the mask used in experiment including dimensions in microns. The figures show an enlargement of the mask middle section. The current tracks pattern is drawn in blue and the voltage contacts pattern is drawn in black color. This mask gives the possibility to pattern tracks of three different widths: 7,5 , and $3 \mu \mathrm{m}$ (zoomed view bottom the figure).

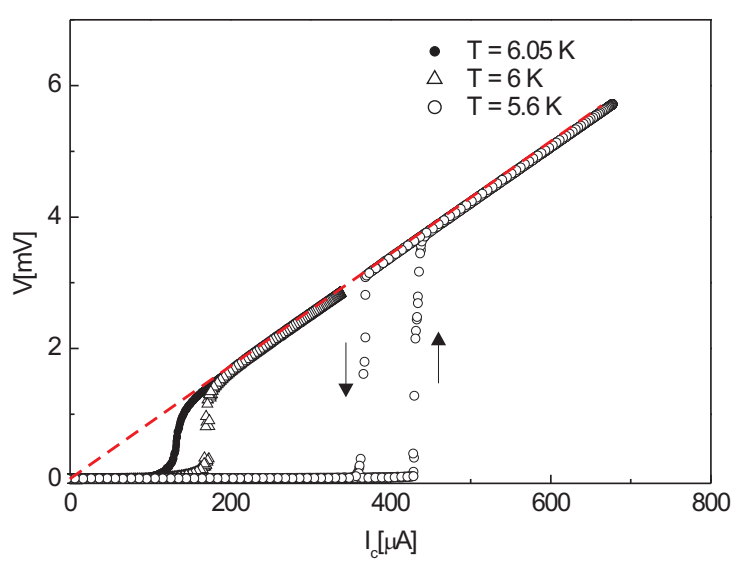

Fig. 2. Current-voltage characteristics, taken at zero field, shows when the normal state is reached. The largest value of current below the voltage onset defines the critical current value. I-V characteristics are shown for three different temperatures, dashed line indicates the normal state resistance.

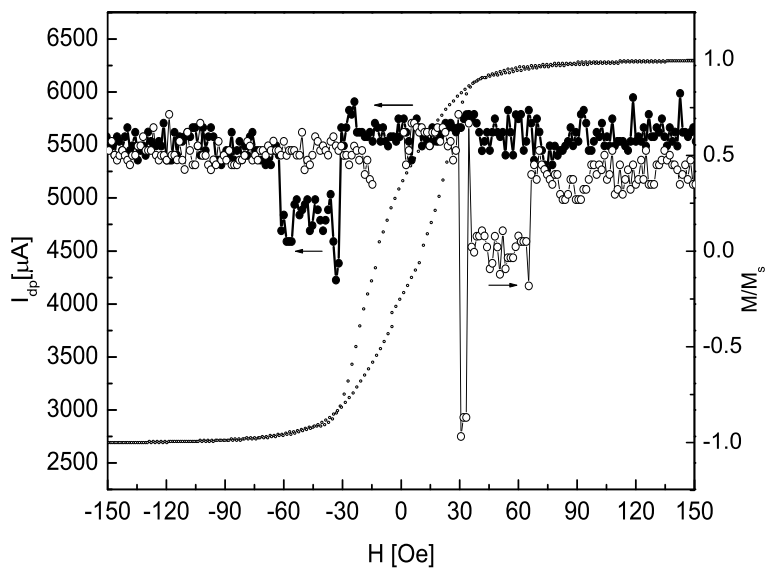

Fig. 3. Critical current vs. in plane magnetic field of a $\mathrm{Nb}(25) / \mathrm{Co}(42)$ sample taken at $T=4.2 \mathrm{~K}$ with $\mathrm{I} \| \mathrm{H}$. Arrows show the field-scan direction. Superimposed is the corresponding hysteresis loop.

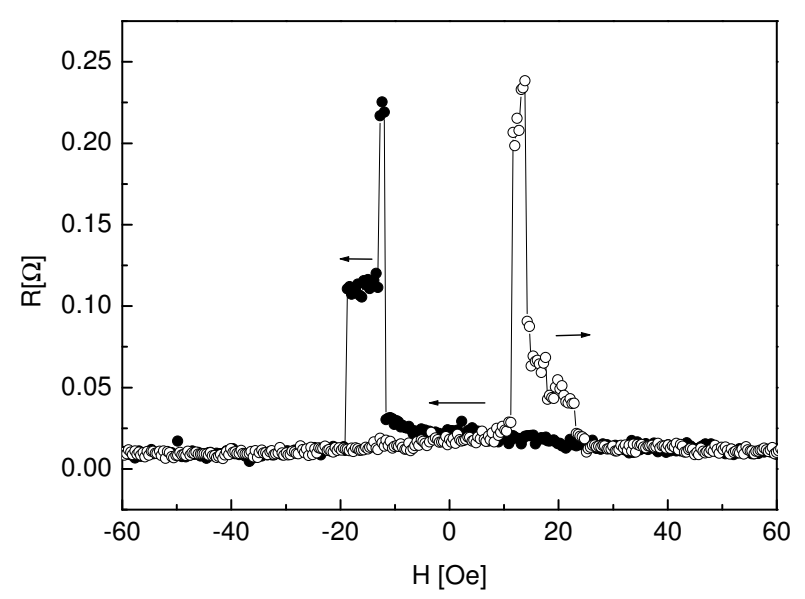

Fig. 4. Resistance as a function of applied field of a patterned sample $\mathrm{Nb}(25) / \mathrm{Py}(50)$, with $\mathrm{I} \| \mathrm{H}$ along the easy anisotropy axes, measured at $6.06 \mathrm{~K}$ where $T_{C} \approx 6.11 \mathrm{~K}$. Arrows show the field scan direction.

current and magnetoresistance measurements (Fig. 4 and 3) the dips and peaks obtained show two defined steps which will be discussed later.

In order to understand these results the I-V characteristics were analyzed close to $T_{C}$ at the dip region. As shown in the inset in Fig. 5 when the applied field is increased from -25 Oe towards -30 Oe where the dip in critical current takes place a voltage onset below the normal critical [12 current value is observed indicating that vortex motion is involved. Because the measurements are made in a Lorentz force free configuration (current parallel to the field) this vortex motion indicates the presence 


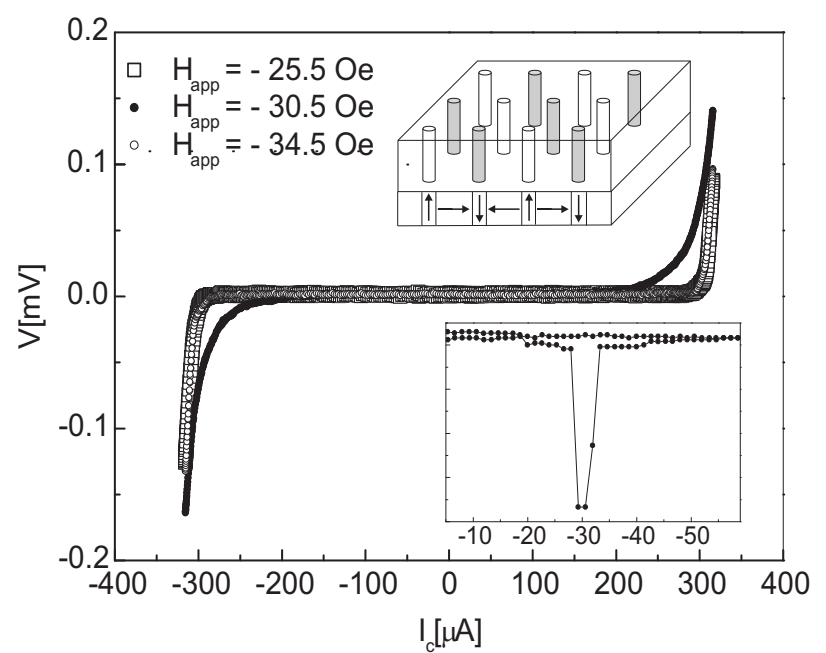

Fig. 5. IV characteristic of $\mathrm{Nb}(25) \mathrm{Co}(50)$ sample taken close to the transition $\approx 0.9 T_{C}$ for different values of field with $\mathrm{I} \| \mathrm{H}$. Motion of vortices is indicated by voltage onset below the normal critical current value. Inset shows vortex-antivortex array distinguished by clear and dark color induced in the superconductor by the Bloch domain structure of the ferromagnet underneath.

of vortices perpendicular to the plane which causes this voltage onset.These are shown in the inset of Fig. 5.

\section{Analysis of Results}

In this letter we present a mechanism by which the experimental observations can be explained. As pointed out previously the magnitude of the dips depends on the ferromagnet's thickness and are only observable beyond a thickness of the ferromagnet $d_{F}>30 \mathrm{~nm}$. This is of the order of thickness where domain walls of the ferromagnet, Co or Py, are thought to gradually mutate from being in plane i.e. Néel domain walls towards the out of plane direction Bloch domain walls. At the range of film thickness; between 35 to $100 \mathrm{~nm}$ the domain structure is formed by cross tie domain walls.

For Co films with a thickness of $50 \mathrm{~nm}$ cross-tie domain walls have been experimentally confirmed using magnetic force microscopy [13. In this case Bloch lines are located periodically with alternating polarity, i.e. pointing outwards or inwards producing high stray fields in this direction, resembling a checkerboard of antiparallel stray fields.

Similar observations for Py films in the domain structure, were found using the Kerr effect, for thicknesses between 30 and $200 \mathrm{~nm}$ [14. The out of plane stray field above a Bloch line can be roughly estimated for $50 \mathrm{~nm}$ Co films based on the observations reported in [13. Here each Bloch line has an approximate width $\delta \approx 0.15-0.3 \mu \mathrm{m}$ with magnetic fields $>2800$ Oe and a magnetic flux between 3-13 $\Phi_{0}$, where $\Phi_{0}$ is the flux quantum. Moreover considering the number of Bloch lines, pointing in the same direction, divided by the interface area, an out of plane effective field of $H_{e f f}^{B D W} \approx \pm 48$ Oe is calculated (this is the maximum value of stray field used later as $B_{o}$ parameter in Eq. (1). Since the lower critical field for a thin $\mathrm{Nb}$ film $H_{c 1}(0)$ in the out of the plane direction is about 10-20 Oe, vortex formation perpendicular to the film is expected.

Furthermore due to the fact that Bloch lines are antiparallel to one another a $2 \mathrm{D}$ vortex-antivortex array pinned by the domain walls must form as shown in the inset Fig. [5] Note also that as the film gets thicker the domains reduce in size, increasing the number of domain walls and Bloch lines that can fit in the same interface area [15] thus augmenting $H_{e f f}^{B D W}$ on the superconductor. These leads to a lower critical current as observed in our experiments after increasing the ferromagnet's thickness.

To have a better understanding of the observed experimental effects the following simple model is employed. As the ferromagnet, initially in the remanent state, lowers its magnetization as result of an in-plane external field $H$, the number of Bloch domain walls $N$ increases augmenting the effective field $H_{\text {eff }}^{B D W}$ (out of plane). This field reaches its maximum value $B_{o}$ at coercive field $H_{c}$ and begins to decrease for $H>H_{c}$. This behavior has been modeled using the following expression:

$$
H_{e f f}^{B D W}=B_{o}\left(1-\left|\frac{M(H)}{M_{S}}\right|\right)
$$

where $M_{S}$ is the saturation magnetization.

Using the value of $H_{c}=20$ Oe for a Co film and a value of $B_{o}=48$ Oe (estimated in the previous paragraph) for Eq. 1. a double peak structure of $H_{\text {eff }}^{B D W}$ is obtained as shown in Fig. 6, This explains the results since when the ferromagnet is in a single domain state the patterned track is in a Lorentz force free configuration (i.e. current parallel to the field). However when the ferromagnet breaks into domains $H_{\text {eff }}^{B D W}$ develops and the patterned track shifts to a Lorentz force configuration where the current is now perpendicular to $H_{\text {eff }}^{B D W}$ resulting in a lower critical current measurement.

\subsection{Discussion}

The absence of any anomalous observations between Cobalt thicknesses $2 \mathrm{~nm}<d_{C o}<30 \mathrm{~nm}$, permits us to conclude that previous observations 4 were probably due to either stray fields from the device geometry or dipole stray fields.

Regarding the observations in magnetoresistance measurements made on $\mathrm{Nb} / \mathrm{Py}$ bilayers [7, the observation of dips is accompanied by peaks in magnetoresistance measurements. These imply the presence of stray fields and thus should be considered in the interpretation of the results. Recently the same author reported additional results on $\mathrm{Nb} / \mathrm{Py}$ bilayers [16, where two different thicknesses of Py were used; 20 and $60 \mathrm{~nm}$. Here small dips and large peaks in magnetoresistance were respectively found. The experiment description indicates that the device geometry includes magnetic voltage contacts; thus is likely that 


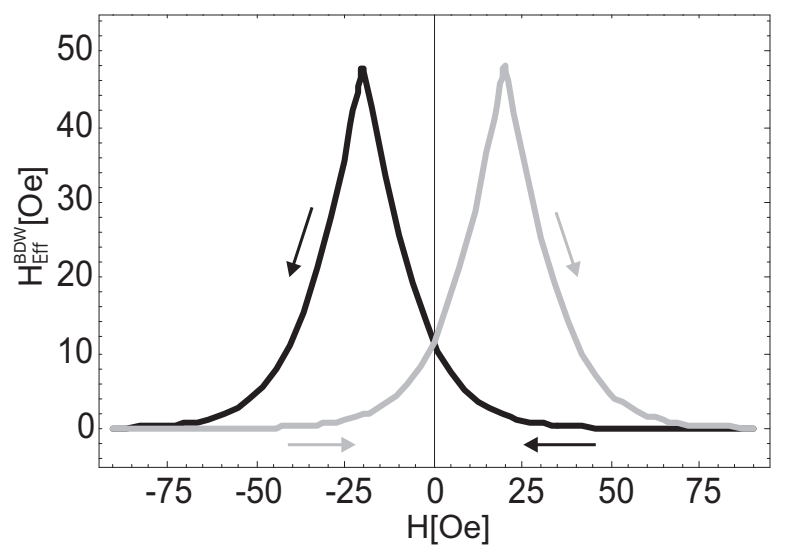

Fig. 6. Stray field from Bloch domain walls is modelled using expression 11. Here $H$ represents the external field applied within the plane of the film while the effective field $H_{e f f}^{B D W}$ develops along the out of plane direction of the film as a double peak structure as a function of applied field.

these dips are due to stray fields. However the large peaks for a Py thickness of $60 \mathrm{~nm}$ are explained by the authors in [16] as the result of Bloch domain wall stray fields. This result is qualitatively similar to the one shown in Fig. 4 and previously described on [17] by the present author. The main difference to our results is the two step structure observed in figures 4 and 3.

Although the previous suggested mechanism in the research investigations 4, 7, 16] (the enhancement of superconductivity at the domain walls i.e. DWS) is probably correct, we believe that an unambiguous experimental evidence of DWS has not yet been successfully produced.

\section{Conclusions}

In summary the dips between coercive field and saturation come as a result of votex-antivortex $2 \mathrm{D}$ lattice formation (perpendicular to plane) which reduces the critical current. These are the result of Bloch lines formed with strong antiparallel stray fields at the domain walls. Increasing the field further and fully magnetizing the sample (where Bloch domain walls are absent) results in a re-entrant behavior to superconductivity as seen in magnetoresistance measurements. Similar properties of so called field-induced superconductivity (FIS) have only been realized by magnetic dots array deposited on top of a superconducting $\mathrm{Pb}$ film [18] and in $50 \mathrm{~nm}$ Niobium films deposited on the single crystal ferrimagnet $\mathrm{BaFe}_{12} \mathrm{O}_{19}$. Both experiments were performed with a magnetization along the perpendicular direction and with an insulator between the superconductor and ferromagnet [19].

Our inference of Bloch domain induced 2D vortexantivortex array in the superconductor makes it possible to experimentally investigate $2 \mathrm{D}$ vortex crystal lattice without the need of superconductors with unconventional pairing symmetry or $\pi$ - Josephson junctions configurations [20].
In both critical and magnetoresistance measurements two sharp steps in the region where dips and peaks are observed indicate an abrupt reconfiguration of the $2 \mathrm{D}$ vortexantivortex array. These two well defined vortex arrangements deserve further investigation, for example using scanning tunnelling microscopy (STM) with resolution on the $\mathrm{nm}$ scale to visualize the vortex dynamics as this could be of great interest for both applied and fundamental research.

Finally, by confining superconductivity to nano dimensions $\leq 2 \xi_{S}$ together with the effect from Bloch domain wall formations makes these structures very sensitive to small magnetic fields. This opens up potential applications such as small field detectors and switching devices.

\section{References}

1. Z. Radović, M. Ledvij, L. Dobrosavljević-Grujić, A.I. Buzdin, and J. R. Clem, Phys. Rev. B 44, 759 (1991).

2. V. V. Ryazanov, V. A. Oboznov, A. Y. Rusanov, A. V. Veretennikov, A. A. Golubov, and J. Aarts, Phys. Rev. Lett. 86, 2427 (2001).

3. J. S. Jiang, D. Davidović, Daniel H. Reich, and C. L. Chien, Phys. Rev. Lett. 74, 314 (1995).

4. R. J. Kinsey, G. Burnell and M. G. Blamire, IEEE Trans. Appl. Supercond., 11, 904, (2001).

5. M. Houset and A. I. Buzdin, Phys. Rev. B 74, 214507, (2006).

6. A. Rusanov, M. Hesselberth, S. Habraken and J. Aarts, Physica C, 404, Issue 1-4, 322 (2004).

7. A. Yu. Rusanov, M. Hesselberth, J. Aarts and A. I. Buzdin, Phys. Rev. Lett. 93, 57002 (2004).

8. V. V. Ryazanov, V. A. Oboznov, A.S. Prokof'ev, and S. V. Dubonos, JETP Lett. 77, 39 (2003).

9. I. S. Burmistrov and N.M. Chtchelkatchev, Phys. Rev. B, 72, 144520 (2005).

10. R. Steiner and P. Ziemann, Phys. Rev. B, 74, 94504 (2006).

11. C. Bell, S. Tursucu and J. Aarts, Phys. Rev. B, 74, 214520 (2006).

12. In most of our meassurements well bellow $T_{C}$ we did not observed flux flow at the dip region but an abrupt reduction of the critical current value. However as shown in Fig. 5 close to $T_{C}$ the pinning barrier is easily overcomed by thermal activation.

13. M. Löhndorf, A. Wadas, H. A. M. van den Berg, and R. Wiesendanger, Appl. Phys. Lett., 68, 25, (1996).

14. S. Methfessel, S. Middelhoek, H. Thomas, IBM Journal, 96, (1959).

15. A. Stankiewiczyz, S. J. Robinsonz, G. A. Gehringzx and V. V. Tarasenkoy, J. Phys: Condens. Matter 9,10191030, (1997).

16. A.Yu. Rusanov, T.E. Golikova, and S.V. Egorov. JETP Lett. 87, 175 (2008)

17. E. J. Patiño, "Study of The Influence of Domain Walls in The Superconductor/Ferromagnet Proximity Effect". Ph.D Thesis [Non-Published], Cambridge University, (2005).

18. M. Lange, M. J. Van Bael, Y. Bruynseraede, and V. V. Moshchalkov, Phys. Rev. Lett. 90, 197006 (2003).

19. Z. Yang, M. Lange, A. Volodin, R. Szymczak and V. V. Moshchalkov, Nature Mat. 3, 793, (2004). 
20. H. Hilgenkamp, Ariando, Henk-Jan H. Smilde, Dave H. A. Blank, G. Rijnders, H. Rogalla, J. R. Kirtley and C. C. Tsuei, Nature 422, 50 (2003). 